\title{
EMERGENCE OF SHIGELLA SONNEI RESISTANT TO KANAMYCIN AND TO NALIDIXIC ACID, WITHOUT EXPOSURE TO THESE DRUGS
}

\author{
MaIR E. M. Thomas AND NaOmi DatTa \\ Epidemiological Research Laboratory, Central Public Health Laboratory, Colindale, London, \\ and Department of Bacteriology, Royal Postgraduate Medical School, London
}

WHATEVER the mechanism of drug resistance in bacteria its emergence in vivo is unexpected, except in response to contact with the relevant or a related antibacterial agent. It is therefore of interest that we have observed what appears to be primary or unselected drug resistance in two family outbreaks of shigellosis in Enfield. The resistance was to kanamycin in one family and to nalidixic acid in the other. Shigella sonnei of colicine type $\mathrm{O}$ was the organism involved in each episode. In the absence of any preceding antibacterial treatment, some but not all of the infected persons in each household consistently excreted apparently pure cultures of a resistant strain.

\section{KANAMYCIN RESISTANCE EPISODE}

In $1966 \mathrm{a}$ girl of $18 \mathrm{mth}$ and her 6-yr-old brother developed gastro-enteritis, and faeces samples from all five members of the family were examined before treatment. Sh. sonnei, colicine type O, was found in every specimen. All the strains were sensitive by paper disk test to chloramphenicol, nalidixic acid, and furazolidone, but resistant to sulphonamide, ampicillin, tetracycline and streptomycin. However, the isolates from four members of this family were also, unexpectedly, resistant to kanamycin and grew well on nutrient agar containing $100 \mu \mathrm{g}$ of this drug per $\mathrm{ml}$. The strain from the mother was kanamycin-sensitive and inhibited by $2 \mu \mathrm{g}$ per $\mathrm{ml}$ in nutrient agar.

Because of this discrepant finding two further faecal specimens were taken from each person in the household, with exactly the same result. Kanamycinresistant Sh. sonnei had not previously been encountered in the Enfield district, or reported elsewhere in Britain, so very detailed enquiries were pursued, but no source of resistant infection could be traced. The family doctor kept careful records and it seems beyond reasonable doubt that kanamycin, neomycin and related drugs had never been administered at home or in hospital to any member of the family.

\section{Bacteriological investigation}

Possible sources of resistance factors. The absence of Sh. sonnei resistant to kanamycin in the community at that time together with the curious partial distribution of drug resistance within the family, suggested that the shigella

Received 21 Feb. 1969; accepted 8 May 1969.

J. MED. MICROBIOL.-VOL. 2 (1969) 
strains might have accepted $\mathrm{R}$ factors from some other enterobacteriaceae present in the bowel, so a search was made for possible donor bacteria. Faecal samples from everyone in the family, taken before treatment, were cultured on MacConkey agar plates with $30-\mu \mathrm{g}$ kanamycin disks. The only kanamycin-resistant organisms found were the four strains of Sh. sonnei already discovered. Possibly a wider range of media or anaerobic incubation might have disclosed more.

Resistance transfer and loss. All the Sh. sonnei strains transferred resistance to Escherichia coli K12 in mixed culture (Watanabe and Fukasawa, 1961). At least two $\mathrm{R}$ factors were present and were classified as $f i+$ or $f-$ (Watanabe et al., 1964; Lawn et al., 1967). One, fi-, was present in all the Sh. sonnei strains and gave resistance to ampicillin, streptomycin, tetracycline and sulphonamides. Kanamycin resistance was determined by a second, $f+$, factor, and the two were transferred independently. The $\mathrm{R}$ factor conferring kanamycin resistance was spontaneously lost at a measurable rate from its $\mathrm{Sh}$. sonnei host: a single kanamycin-resistant colony was grown in antibiotic-free broth for $4 \mathrm{hr}$ and dilutions were spread on nutrient agar plates to obtain single colonies. When these were tested by replicating to kanamycin-containing agar, four kanamycinsensitive colonies were identified. They had lost the $f i+\mathrm{R}$ factor but retained the $f i$ - one, and resembled exactly the kanamycin-sensitive strains isolated from the mother of the family.

Mutation to resistance. Kanamycin-resistant variants were not recovered from serial subcultures of the initial kanamycin-sensitive strain, nor from those which had spontaneously lost kanamycin-resistance in the laboratory.

Growth rates. When the resistant and the sensitive strains were grown in parallel, in broth, no obvious advantage in growth rate was evident for either. When kanamycin-resistant strains were grown in mixed culture with kanamycinsensitive ones the culture became predominantly kanamycin-resistant, no doubt because the resistance was carried on an infectious $\mathrm{R}$ factor.

\section{NALIDIXIC ACID RESISTANCE EPISODE}

In 1967 all seven children of a family developed dysentery during a weekend. Three of them attended a primary school at which four cases of dysentery due to Sh. sonnei, colicine type O, had recently occurred. The strains in these four school infections were sensitive to streptomycin, tetracycline, chloramphenicol, neomycin, kanamycin, nalidixic acid and furazolidone, but resistant to sulphonamide and ampicillin. The patients were treated with furazolidone. Sh. sonnei type $\mathrm{O}$ with the above antibiotic-sensitivity pattern was isolated from three members of the family, but cultures from another two of the children, though also of type $O$, differed unexpectedly in being resistant to nalidixic acid. They were not inhibited by disks containing $30 \mu \mathrm{g}$ of the drug, and grew well on agar containing $40 \mu \mathrm{g}$ per ml. Nalidixic acid-resistant Sh. sonnei had not been seen previously. From the remaining two children and the parents no shigellae were isolated.

Two further faecal samples were taken from each member of the family, with identical results. The family had been for many years under the care of a 
general practitioner who kept careful records, and a detailed enquiry was made about previous drug treatment. Nalidixic acid had never been previously administered to any member of the family.

At this juncture nalidixic acid was administered to the whole family. Sh. sonnei continued to be excreted by the two children with resistant infections, but was not subsequently isolated from the rest of the family.

\section{Bacteriological investigations}

Possible sources of resistance factors. The novelty of Sh. sonnei strains resistant to nalidixic acid and their discovery in a household where some persons were excreting sensitive strains suggested that the resistance might be determined by an $\mathrm{R}$ factor, although resistance to this drug has never yet been found to be transmissible. A search was therefore made for possible donor bacteria. Faeces from every member of the household, taken before treatment, were cultured on MacConkey agar plates with nalidixic acid disks. The only nalidixic acid-resistant organisms found were the Sh. sonnei already described, and in one uninfected adult, a faecal streptococcus.

Tests for resistance transfer and for mutation to resistance. All the Sh. sonnei strains from this second household carried a transmissible $\mathbf{R}$ factor conferring sulphonamide resistance, but no transfer of resistance to ampicillin or nalidixic acid was found. Although nalidixic acid-resistance has never been reported to be transferable, unstable resistance was found in Shigella flexneri (Lewis, 1967). No spontaneous loss of nalidixic acid-resistance was observed in our Sh. sonnei strains when 1500 colonies were tested by replica plating, but resistant mutants could be recovered from single-colony isolates of the nalidixic acidsensitive Sh. sonnei strains. About $10^{9}$ cells, grown in drug-free broth from a small inoculum (c. 10 cells) of sensitive Sh. sonnei, were plated on nutrient agar containing $25 \mu \mathrm{g}$ nalidixic acid per $\mathrm{ml}$; three resistant colonies developed, which resembled the naturally occurring nalidixic acid-resistant strains from the same household except that they had a slightly lower level of resistance. Sensitive strains were inhibited by $1.25 \mu \mathrm{g}$ nalidixic acid per $\mathrm{ml}$ in nutrient agar plates, but the three selected resistant mutants were inhibited at $40 \mu \mathrm{g}$ per ml, and the naturally occurring resistant strains only by $80 \mu \mathrm{g}$ per $\mathrm{ml}$.

Growth rates. When the resistant and sensitive strains were grown in parallel in broth, and their growth measured turbidimetrically, no obvious advantage in growth rate was seen in either. Nalidixic acid-resistant strains were grown in mixed broth cultures with sensitive strains from the same family outbreak. Viable counts were made on nutrient agar with and without incorporated nalidixic acid $(25 \mu \mathrm{g}$ per $\mathrm{ml})$ with repeated subculture of the mixtures through over 100 bacterial generations. The proportion of resistant to total organisms in the mixtures remained almost constant.

\section{Discussion}

Primary resistance of Sh. sonnei to kanamycin is rare (Davies, Farrant and Tomlinson, 1968a and $b$; Aoki, 1968) and resistance to nalidixic acid has not 
been reported previously except after treatment with the drug (Couston, 1966; Fernandez, 1966). The circumstances in which resistance to kanamycin and to nalidixic acid were observed in the episodes described are interesting.

In the two household outbreaks of dysentery, each studied before the start of treatment, certain individuals consistently excreted novel resistant strains of Sh. sonnei, whereas others excreted sensitive strains of the same colicine type. Neither drug had ever been administered to either family. No other enterobacteriaceae resistant to either drug were found in any of the samples of faeces examined. There was, therefore, no evidence of selection of resistance by exposure to the drug involved, or for the acquisition of transferable resistance factors within the gut, nor was there any ascertainable community reservoir of resistant strains. In fact such strains had not been encountered before. They appeared to arise within the untreated households.

An increase in the proportion of drug-resistant strains of Sh. sonnei during the last decade has resulted from the acquisition of $\mathrm{R}$ factors (Farrant and Tomlinson, 1966; Davies et al., 1968a); indeed extra resistance genes were shown to be acquired during the course of at least 8.5 per cent. of family outbreaks (Davies et al., 1968b), but the increase has been thought to be due not only to the presence of $R$ factors in the bowel flora but also to the selective effect of exposure to the drugs concerned, particularly sulphonamides, ampicillin, streptomycin and tetracycline. Conversely certain substances, particularly acridine dyes and among them such drugs as mepacrine, are known to "cure" bacteria of such extrachromosomal genetic elements as R factors (Mitsuhashi, Harada and Kameda, 1961; Lebek, 1967; Tomoeda et al., 1968). The possible interactions are complex. In our cases the kanamycin-resistance factor was unstable and spontaneous loss took place in the laboratory. Its origin was not discovered.

The nalidixic acid-resistance found in the second family could not be explained either. Single-step mutation of Sh. sonnei cultures to a significant degree of resistance occurs with nalidixic acid, but in the absence of some selective advantage, such as exposure to the drug or an advantage in growth rate, the supplanting of the drug-sensitive parent population would not be expected. Yet no difference in growth rates was found, and neither our patients nor the preceding cases at their school had been treated with nalidixic acid. Variation in genetic qualities acquired from the environment, such as transmissible resistance or colicinogeny factors, have been described (Davies et al., 1968b). This study shows that these and other variations may be encountered even within one household where the infection probably originated from a single source.

\section{SUMMARY}

Sh. sonnei resistant to kanamycin in one household and, in another family, to nalidixic acid, is described. Resistance of this organism to these drugs is uncommon. Some individuals within each family excreted sensitive strains of the shigella. There was no history of previous exposure to either drug and the source of the resistant strains is unknown. As far as we know these strains 
neither arose from nor spread to the community. Kanamycin-resistance was determined by a transferable factor; no transfer of nalidixic acid-resistance could be demonstrated. The supplanting of the prevailing sensitive strains by new drug-resistant strains of $S h$. sonnei is unexplained in both families.

\section{REFERENCES}

AOKI, Y.

1968. Arch. Immunol. Thér. exp., 16, 303.

Couston, T. A 1966. Med. Offr, 115, 147.

Davizs, JoAn R., Farrant, W. N., AND 1968a. J. Hyg., Camb., 66, 471. TOMLINSON, A. J. H.

" " $\quad$ ",$\quad$ " 1968b. Ibid., 66, 479.

Farrant, W. N., AND Tomlinson, A. J. 1966. Ibid., 64, 287.

$\mathrm{H}$.

FERNANDEZ, L. A.

1966. J. Kansas Med. Soc., 67, 359.

Lawn, A. M., Meynell, Elinor, Mey- 1967. Nature, Lond., 216, 343.

NELl, G. G., AND DATTA, NAOMI

LEBEK, G. . . . . . . . .

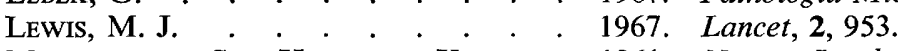

Mitsuhashi, S., Harada, K., AND 1961. Nature, Lond., 189, 947.

KAMEDA, M.

Tomoeda, M., Inuzuka, M., Kubo, N., 1968. J. Bact., 95, 1078. AND NaKamura, $S$.

Watanabe, T., AND Fukasawa, T. . . 1961. Ibid., 81, 669.

Watanabe, T., Nishida, H., Ogata, C., 1964. Ibid., 88, 716.

ARAI, T., AND Sato, S.

\section{Addendum}

In a recently published paper (Smith, H. W., 1969, Lancet, 1, 1174), the author reports the isolation in vitro of a nalidixic acid-resistant mutant of the resident Escherichia coli of a person, who was then given $1 \mathrm{ml}$ of a broth culture of the mutant by mouth. No nalidixic acid was administered to select for resistance in vivo, but the resistant mutant nevertheless colonised the intestine and constituted the bulk of $E$. coli colonies isolated from the person over the next two years, presumably because it had some unexplained selective advantage in the intestine. 